\title{
A COUNTEREXAMPLE CONCERNING INSEPARABLE FIELD EXTENSIONS
}

\author{
JAMES KEVIN DEVENEY
}

\begin{abstract}
Let $K \supseteq M \supseteq k$ be a chain of fields of characteristic $p \neq 0$ where $K$ is separable over $M$ and $M$ is purely inseparable over $k$. Recently it has been shown that if $K$ has a separating transcendency basis over $M$ or if $M$ is of bounded exponent over $k$, then $K=M \otimes_{k} S$ where $S$ is separable over $k$. This note presents an example to show that, in general, no such $S$ need exist.
\end{abstract}

Throughout, we consider a chain of fields $K \supseteq M \supseteq k$ of characteristic $p \neq 0$ where $K$ is separable over $M$ and $M$ is purely inseparable over $k$. Recent papers [1] and [2], have examined the question of when $K$ can be expressed as $M \otimes_{k} S$ where $S$ is a separable extension of $k$. It has been shown that if $M$ is of bounded exponent over $k$ [1, Theorem 5], or if $K$ has a separating transcendency basis over $M$ [2, Lemma 4], then $K=M \otimes_{k} S$ for some $S$. The purpose of this note is to provide an example to show that, in general, no such $S$ exists. Necessarily, $M$ will be of unbounded exponent over $k$ and $K$ will not have a separating transcendency basis over $M$.

ExAmple 1. Let $P$ be a perfect field of characteristic $p \neq 0$ and let $\left\{x_{1}\right.$, $\left.x_{2}, \ldots, x_{n}, \cdots\right\}$ be an algebraically independent set over $P$. Set

$K=P\left(x_{1}, \ldots, x_{n}, \cdots\right)$,

$M=P\left(x_{1} x_{2}^{p}, x_{2} x_{3}^{p}, \ldots, x_{n} x_{n+1}^{p}, \cdots\right)$,

$k=P\left(x_{1}^{p} x_{2}^{p^{2}}, x_{2}^{p^{2}} x_{3}^{p^{3}}, \ldots, x_{n}^{p^{n}} x_{n+1}^{p^{n+1}}, \cdots\right)$.

Since $\left(x_{n} x_{n+1}^{p}\right)^{p^{n}} \in k$ for all $n, M$ is purely inseparable over $k .\left\{x_{1} x_{2}^{p}\right.$, $\left.x_{2} x_{3}^{p}, \ldots, x_{n} x_{n+1}^{p}, \cdots\right\}$ is a $p$-basis for $M$ and remains $p$-independent in $K$, so $K$ is separable over $M$. Moreover, elementary calculations show $\left\{x_{1} x_{2}^{p}, \ldots, x_{n} x_{n+1}^{p}, \cdots\right\}$ is actually a $p$-basis for $K$, and thus $K$ is relatively perfect over $M$, i.e. $K=M\left(K^{p}\right)$. We now assume there exists a field $S$ separable over $k$ such that $K=M \otimes_{k} S$.

LEMMA 2. $S$ is relatively perfect over $k$.

Proof. Recall that $K=M\left(K^{p}\right)$. Since we are assuming $K=M(S), K^{p}$ $=M^{p}\left(S^{p}\right)$, and so $K=M\left(M^{p}\right)\left(S^{p}\right)=M\left(S^{p}\right)$. Thus $K=M \otimes_{k} k\left(S^{p}\right)$ and we must have $S=k\left(S^{p}\right)$.

Now since $S$ is relatively perfect over $k, S=k\left(S^{p^{n}}\right) \subseteq k\left(K^{p^{n}}\right)$ for all $n$. Thus $S \subseteq \cap k\left(K^{p^{n}}\right)$.

Lemma 3. $\cap k\left(K^{p^{n}}\right) \subseteq P\left(x_{1}^{p}, x_{2}^{p^{2}}, \ldots, x_{n}^{p^{n}}, \cdots\right)=\bar{k}$.

Proof. Since $\bar{k} \supseteq k, \cap k\left(K^{p^{n}}\right) \subseteq \cap \bar{k}\left(K^{p^{n}}\right)$. Since

Received by the editors November 18, 1974.

AMS (MOS) subject classifications (1970). Primary $12 \mathrm{~F} 15$. 


$$
K=\bar{k}\left(x_{1}\right) \otimes_{\bar{k}} \bar{k}\left(x_{2}\right) \otimes_{\bar{k}} \cdots \otimes_{\bar{k}} \bar{k}(x) \otimes_{\bar{k}} \cdots,
$$

$\cap \bar{k}\left(K^{p^{n}}\right)=\bar{k}$, and the lemma is established.

We now have $S \subseteq \cap k\left(K^{p^{n}}\right) \subseteq \bar{k}$. To show no such $S$ exists it suffices to show $M(\bar{k}) \neq K$.

$$
\begin{aligned}
M(\bar{k}) & =P\left(x_{1} x_{2}^{p}, \ldots, x_{n} x_{n+1}^{p}, \cdots\right)\left(x_{1}^{p}, x_{2}^{p^{2}}, \ldots, x_{n}^{p^{n}}, \cdots\right) \\
& =P\left(x_{1} x_{2}^{p}, \ldots, x_{n} x_{n+1}^{p}, \cdots\right)\left(x_{1}^{p}\right)=M\left(x_{1}^{p}\right) .
\end{aligned}
$$

$P\left(x_{1}, \ldots, x_{n}\right)$ is algebraic over $P\left(x_{1}, x_{1} x_{2}^{p}, \ldots, x_{n-1} x_{n}^{p}\right)$, and hence both fields have the same transcendence degree $n$ over $P$, which means that $x_{1}$, $x_{1} x_{2}^{p}, \ldots, x_{n-1} x_{n}^{p}$ are algebraically independent over $P$. Since this is true for all $n$, the set $\left\{x_{1}, x_{1} x_{2}^{p}, x_{2} x_{3}^{p}, \cdots\right\}$ is algebraically independent over $P$, and hence $x_{1}$ is transcendental over $M=P\left(x_{1} x_{2}^{p}, x_{2} x_{3}^{p}, \cdots\right)$. By Luroth's theorem $M\left(x_{1}^{p}\right) \subsetneq M\left(x_{1}\right) \subseteq K$. Thus no such $S$ can exist.

\section{REFERENCES}

1. N. Heerema and H. F. Kreimer, Modularity vs. separability for field extensions, Canad. J. Math. (to appear).

2. N. Heerema and D. Tucker, Modular field extensions, Proc. Amer. Math. Soc. 53 (1975), $301-306$.

Department of Mathematical Sciences, Virginia Commonwealth University, RichMOND, VIRGINIA 23284 